\title{
Early aortic valve cusp rupture in relapsing polychondritis
}

\author{
D A S Marshall, R Jackson, A P Rae, H A Capell
}

\begin{abstract}
Aortic regurgitation associated with relapsing polychondritis usually occurs late in the disease as a result of aortic root dilatation.

A case where aortic regurgitation occurred early and was due to cusp rupture with a normal aortic root is reported. The patient required urgent aortic valve replacement within six weeks of developing a murmur despite apparent control of inflammation with immunosuppressive treatment.

The possibility of cusp rupture with sudden haemodynamic deterioration should be considered in patients with relapsing polychondritis who develop aortic regurgitation.
\end{abstract}

\section{Case report}

A 57 year old woman presented to her family doctor with a four month history of nasal stuffiness and xerostomia. She was initially treated with nasal cromoglycate and steroid inhalers but within four weeks developed dry eyes, temporomandibular discomfort, and noticed a 'bump' on her nose. There was no history of any previous illnesses, in particular arthritis, rheumatic fever, or other cardiac disease.

Examination showed bilateral conjunctivitis, a tender right temporomandibular joint, and a 'saddle nose' (fig 1) with depression of the cartilage in the region of the nasal ridge. Her temperature was normal, pulse was 90 beats/ min in sinus rhythm with a supine blood pressure of $150 / 60 \mathrm{mmHg}$. Auscultation showed $\bar{a}$ grade III/VI early diastolic murmur, consistent with aortic regurgitation.

Initial investigations showed haemoglobin 98 $\mathrm{g} / \mathrm{l}$ with marked rouleaux formation, total white cell count $15.9 \times 10^{9} / 1$ with $92 \%$ neutrophilia and platelet count $519 \times 10^{9} / 1$. Erythrocyte sedimentation rate was $94 \mathrm{~mm} / 1 \mathrm{st} \mathrm{h}$. Blood urea and electrolytes were normal; plasma protein electrophoresis showed a polyclonal rise in $\gamma$ and $\alpha_{2}$ globulins. Chest radiograph and standard 12-lead electrocardiogram were both normal. Bone marrow aspirate showed reactive hypercellular changes with a mild plasmacytosis. Serial blood cultures were negative. Rheumatoid factor was weakly positive at a titre of $1 / 64$, but titres to antinuclear factor, anti-Ro, anti-La, anti-RNP, anti-Sm, and DNA binding capacity were negative. Skeletal muscle biopsy and Kveim test were also negative.

No definite diagnosis was made at this stage and treatment was started empirically with oral prednisolone $40 \mathrm{mg}$ daily. Forty eight hours later, however, she was readmitted with acute pulmonary oedema and oliguria and was subsequently transferred to Glasgow Royal Infirmary.

Physical examination showed her to be tachypnoeic, cyanosed, and pale. Temperature was $36^{\circ} \mathrm{C}$, pulse 140 beats $/ \mathrm{min}$, and blood pressure $160 / 20 \mathrm{mmHg}$. The jugular venous pulse was raised by $6 \mathrm{~cm}$, the apex beat was displaced and lifting with a palpable third heart sound. Auscultation disclosed a loud early diastolic murmur and gallop rhythm. Apex phonocardiography was not performed. Lung fields were congested with bilateral pleural effusions clinically obvious. Chest radiography confirmed pulmonary oedema and pleural effusions with cardiomegaly. Electrocardiogram showed sinus tachycardia with left ventricular strain pattern. The severe aortic valve insufficiency was thought sufficient to cause acute cardiac dilatation.

She was treated with high dose diuretic therapy, and the clinical features of acute pulmonary oedema resolved. Further investigations were as follows: serum creatinine $\mathbf{1 7 0}$ $\mu \mathrm{mol} / \mathrm{l}$, blood urea $34 \cdot 2 \mathrm{mmol} / \mathrm{l}$, erythrocyte sedimentation rate $120 \mathrm{~mm} / 1 \mathrm{st} \mathrm{h}$. Urine analysis

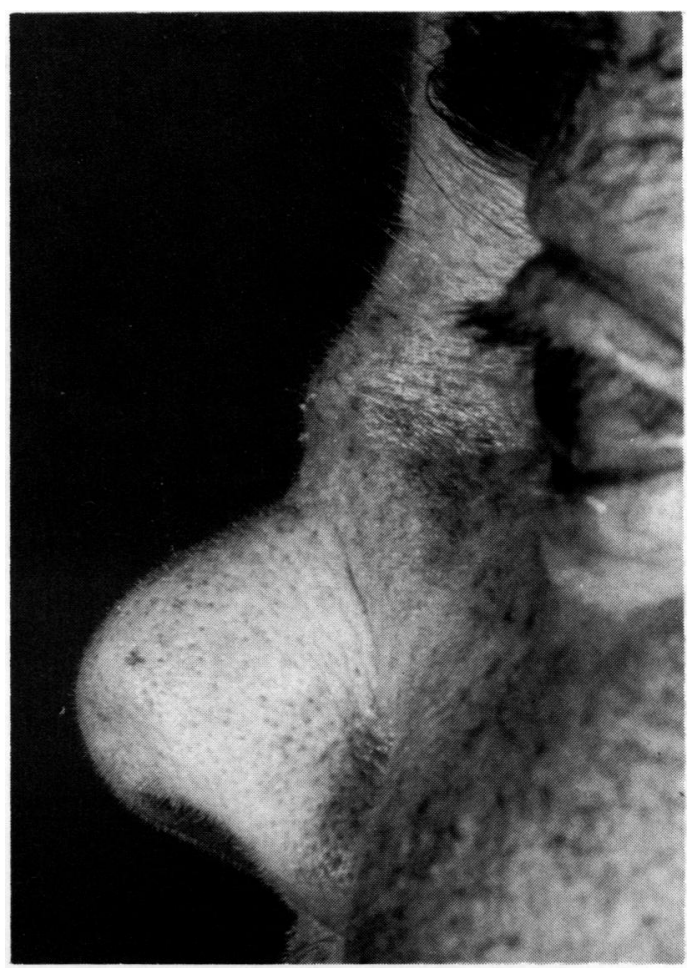

Figure 1 Typical appearance of saddle nose deformity. 
showed both blood and protein, but blood cultures were again repeatedly negative.

Echocardiography showed a left ventricle dilated by hyperdynamia (left ventricle end diastolic diameter $64 \mathrm{~mm}$ ) (fig 2) with a normal sized aortic root and normally mobile tricuspid aortic valve. No vegetations were seen. The anterior mitral leaflet showed pronounced fluttering with early closure, in keeping with severe aortic regurgitation. An Austin Flint murmur was not evident clinically. Continuous wave Doppler examination confirmed severe aortic regurgitation. Percutaneous renal biopsy was performed, which showed a severe, focal, necrotising crescentic glomerulonephritis. Although antineutrophil cytoplasmic antibody titres were negative, antibodies to cartilage (against type II collagen) were found at a significant titre of $1 / 16$. These features, together with the clinical appearance, were consistent with a diagnosis of relapsing polychondritis.

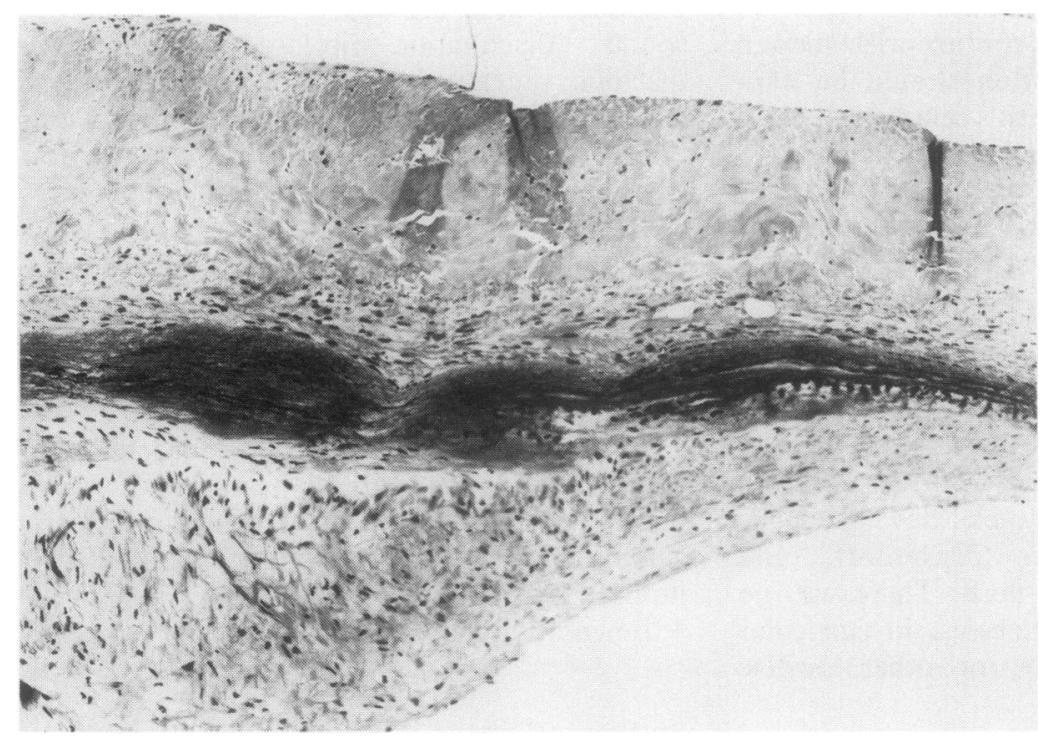

Figure 2 Histopathology of affected valve cusp showing line of shearing with hyaline degeneration, chronic inflammatory infiltrate, and oedema.

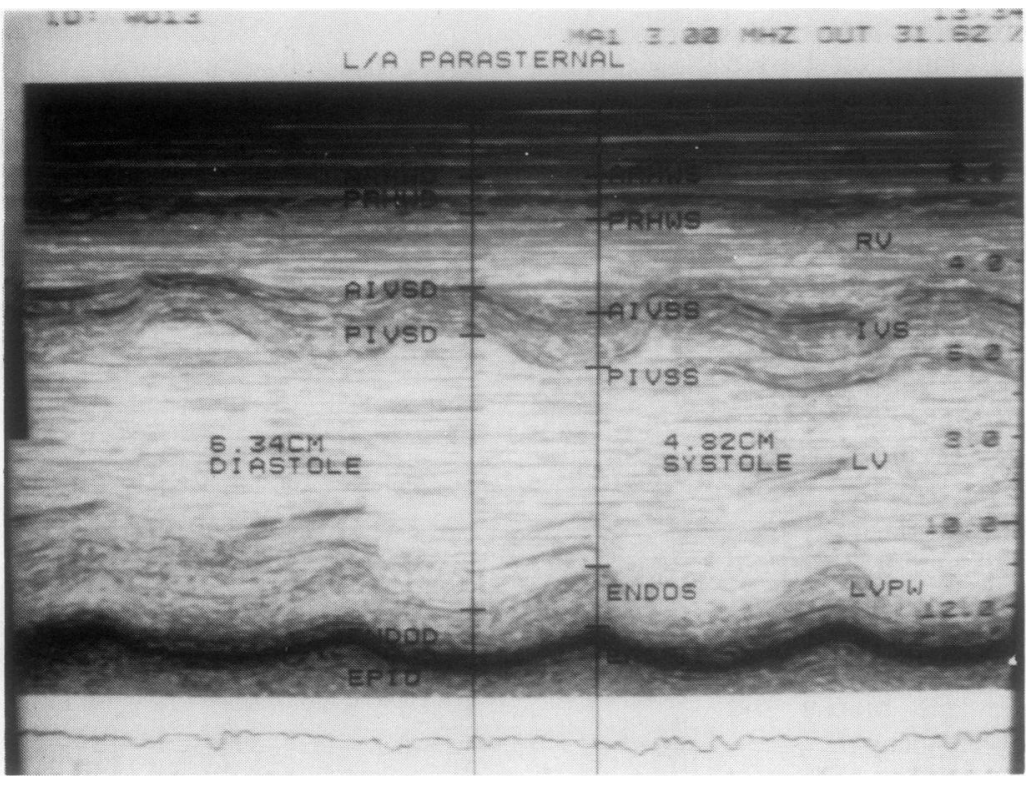

Figure 3 M mode echocardiogram showing dilated left ventricle.
She was given immunosuppressive treatment daily with cyclophosphamide $3 \mathrm{mg} / \mathrm{kg}$ and prednisolone $60 \mathrm{mg}$ orally. This resulted in an improvement in the indices of inflammation. In particular the erythrocyte sedimentation rate fell from 120 to $30 \mathrm{~mm} / 1 \mathrm{st} \mathrm{h}$ and $\mathrm{C}$ reactive protein from 79 to $10 \mathrm{mg} / \mathrm{l}$ over 12 days. Serum creatinine returned to normal.

She was discharged but reviewed weekly as an outpatient. Her anti-inflammatory drugs were gradually reduced with a view to elective valve surgery. This was, however, pre-empted after three weeks by a further episode of left ventricular failure necessitating admission. At that time the erythrocyte sedimentation rate was $115 \mathrm{~mm} / \mathrm{lst} \mathrm{h}$ and $\mathrm{C}$ reactive protein was markedly raised at $110 \mathrm{mg} / \mathrm{l}$. Thirty six hours after admission she developed a pale, pulseless, and tender left leg requiring emergency superficial femoral artery embolectomy. A $2 \mathrm{~cm}$ solid thrombus was removed.

Postoperatively her condition deteriorated with progressive renal and hepatic impairment together with worsening pulmonary oedema. Echocardiography now showed an obvious deficiency of the aortic valve right coronary cusp with a markedly dilated left ventricle (end diastolic diameter now $88 \mathrm{~mm}$ ). Emergency aortic valve replacement was then undertaken. At operation the right coronary cusp was found to be torn along its full length with evidence of an acute inflammatory reaction at the base of the valve. The other valve cusps seemed normal and the aortic root was of normal calibre and showed no inflammation. No infective vegetations or thrombus were found. The value was excised and a $23 \mathrm{~mm}$ Carpentier-Edwards porcine bioprosthesis was fitted.

Histopathology of the affected valve cusp showed adherent fibrin on the torn exposed surface with a dense leucocyte cellular infiltrate and marked neovascularisation. A line of shearing with marked hyaline degeneration and surrounding oedema was evident (fig 3). Left and non-coronary cusps of the aortic valve were normal and culture for infection was negative.

Immediate postoperative recovery was uncomplicated with rapid resolution of renal and hepatic impairment. She was discharged on the 23rd postoperative day while continuing with immunosuppressive treatment. The most recent echocardiogram showed a normally functioning bioprosthesis and only minimal left ventricular dilatation. The patient remains well 13 months after operation and treatment continues with prednisolone $4 \mathrm{mg} /$ day only. She has had no relapses of polychondritis and renal function is normal.

\section{Discussion}

Relapsing polychondritis is an uncommon disorder characterised clinically by recurrent episodes of inflammation affecting any cartilaginous structures, synovial membranes, the cardiovascular system, and eyes. The disease is thought to have an immunological mechanism with serum autoantibodies to type II collagen present in up to two thirds of cases. This autoantibody is not specific to relapsing poly- 
chondritis but is also found in rheumatoid arthritis, juvenile chronic arthritis, systemic lupus erythematosus, and osteoarthrosis.

Cardiovascular consequences of the recurring inflammatory process have been noted since the early case reports, the commonest of which is aortic aneurysm formation. ${ }^{1-3}$ The first description of aortic insufficiency was by Yamazaki in $1966 .{ }^{4} \mathrm{~A}$ number of studies since have described the pathological features of aortitis with late secondary aortic ring dilatation and aortic aneurysm formation as the primary cause of valvular regurgitation..$^{5-7}$ Histopathological studies have shown evidence of collagen and elastic tissue destruction of the valve cusps. ${ }^{8}$ This deformity of the valves, however, was thought to represent mechanical stress secondary to the dilatation of the aortic root itself rather than a primary condition of the valve leaflets. In this presentation the evidence is more suggestive of a primary involvement of the valve cusp. In our patient the sudden deterioration in clinical condition and concomitant peripheral embolus raised the possibility of rupture of the aortic valve cusp with a fragment of valve tissue entering the systemic circulation, though histology of the embolus showed only thrombus. The clot might well have arisen from the dilated left ventricle, though at operation no mural thrombus was seen. Peripheral embolism is a rare complication of acute aortic regurgitation and, as far as we know, has not been previously reported in this condition.

Previous reports of aortic regurgitation complicating relapsing polychondritis have quoted this as a late consequence of the disease, occurring two to seven years after presentation, particularly in patients with frequent relapses. ${ }^{910}$ Our patient showed early evidence of aortic insufficiency only two months after presenting to the general practitioner with nasal stuffiness. The cardiac dilatation and acute left ventricular failure which rapidly developed might have been precipitated by the introduction of steroid treatment. The concomitant acute glomerulonephritis suggests a severe, first onset of the disease process with circulating immune complexes accounting for the crescent formation, though these were not examined for in this case. Acute glomerulonephritis has been described as occurring in relapsing polychondritis in up to $10 \%$ of cases and is associated with a poorer prognosis.

\section{Conclusion}

The case demonstrates severe aortic regurgitation and left ventricular failure occurring early in the course of relapsing polychondritis due, not to aortic root dilatation, but to direct involvement of the aortic valve cusp itself.

1 Pearson C M, Klein H M, Newcomer V D. Relapsing polychondritis. $N$ Engl f Med 1960; 263: 51-8.

2 Hainer J W, Hamilton $\mathbf{G}$ W. Aortic abnormalities in relapsing polychondritis. $N$ Engl I Med 1969; 280: 1166-8.

3 Hughes R AC, Berry CL, Seifert M. Relapsing polychondritis. Qf Med 1972; 163: 363-80.

4 Yamazaki N, Yawata K, Kamiya H, Kimura E. Case of relapsing polychondritis associated with aortic insufficiency. relapsing polychondritis associ.

$7 p$ Heart $\mathcal{F} 1966 ; 6:$ : 188-95.
5 Esdaile J, Hawkin D, Gold P. Vascular involvement in relapsing polychondritis. Can Med Assoc $\mathcal{J}$ 1977; 116: 1019-22.

6 Mestres C A, Igual A, Botey A, Revert L, Murta $M$. Relapsing polychondritis with glomerulonephritis and severe aortic insufficiency surgically treated with success. Severe aortic insufficiency surgically trea.
Thorac Cardiovase Surg 1983; 31: 307-9.

7 Manna R, Annese V, Ghirlanda G, et al. Relapsing polychondritis with severe aortic insufficiency. Clin Rheumatol 1985; 4: 474-80.

8 Alexander C S, Derr R F, Sako Y. Abnormal amino acid and lipid composition of aortic valve in relapsing polychondritis. Am 7 Cardiol 1971; 28: 337-41.

9 Michet C J, McKenna C H, Luthra H S, O'Fallon W M. Relapsing polychondritis: survival and predictive role of early disease manifestations. Ann Intern Med 1986; 104: 74-8.

10 Sohi G S, Desai M, Ward W W, Flowers N C. Aortic cusp involvement causing severe aortic regurgitation in a case of relapsing polychondritis. Cathet Cardiovasc Diagn 1981; 7: 